\title{
Heart Rate Monitoring Sensor Based on Singlemode-Multimode-Singlemode Fiber
}

\author{
Ninik IRAWATI ${ }^{1,2 *}$, Agus Muhamad HATTA ${ }^{1 *}$, \\ Yoseph Gita Yhun YHUWANA ${ }^{3}$, and SEKARTEDJO ${ }^{1}$ \\ ${ }^{1}$ Department of Engineering Physics, Institute Teknologi Sepuluh Nopember, Surabaya 60111, Indonesia \\ ${ }^{2}$ Aston Institute of Photonics Technologies, Aston University, Aston Express Way, B4 \& ET Birmigham, United Kingdom \\ ${ }^{3}$ Department of Physics, Airlangga University, Surabaya 60115, Indonesia \\ *Corresponding authors: Ninik IRAWATI and E-mail: ninikirawati.pre@gmail.com and \\ Agus Muhamad HATTA amhatta@ep.its.ac.id
}

\begin{abstract}
The singlemode-multimode-singlemode (SMS) fiber structure for a heart rate monitoring is proposed and developed. An artificial electrocardiogram (ECG) signal is used to simulate the heart pulse at different rates ranging from 50 beats per minute (bpm) to $200 \mathrm{bpm}$. The SMS fiber structure is placed at the center of a loudspeaker and it senses the vibration of the pulse. The vibration of the pulse signal applied to the SMS fiber structure changes the intensity of the optical output power. The proposed sensor shows a linear frequency of the heart rate sensing range that matches well with the relevant heart rate from the artificial ECG. This work shows the capability of the SMS fiber structure monitoring the heart rate frequencies for a long term, high stability realization, and reproducibility, and being suitable for the observation in hospitals as well as in other environments.
\end{abstract}

Keywords: Heart rate monitoring; fiber optic sensor; SMS fiber; singlemode-multimode-singlemode fiber; hear rate sensor

Citation: Ninik IRAWATI, Agus Muhamad HATTA, Yoseph Gita Yhun YHUWANA, and SEKARTEDJO, "Heart Rate Monitoring Sensor Based on Singlemode-Multimode-Singlemode Fiber," Photonic Sensors, 2020, 10(2): 186-193.

\section{Introduction}

Heart rate monitoring is one of the key measurements in order to determine the fitness level of a person. For example, once the resting body starts to work, the heart rate will increase. The fastest heart rate can occur when the body has a high intensity of work-out. The heart rate is correlated to the cardiovascular outcomes as well as in patients with pulmonary, cerebrovascular, renal diseases, cancer, and erectile dysfunction [1]. The heart rate of adults at normal conditions ranges from
60 beats per minute (bpm) to $100 \mathrm{bpm}$.

Monitoring of heart rate during diathermy conditions is almost impossible due to electromagnetic disturbances from the electrocardiogram (ECG) signal [2]. Other than that, monitoring the heart rate during magnetic resonance imaging (MRI) and X-ray procedures is also difficult to be done. The most common problem encountered during the ECG is the variation of electrode polarization and impedance in the electrode interface. In addition, the paste and electrodes are common complication that can cause

Received: 20 May 2019 / Revised: 14 September 2019

C) The Author(s) 2019. This article is published with open access at Springerlink.com

DOI: $10.1007 / \mathrm{s} 13320-019-0572-7$

Article type: Regular 
skin irritation. Therefore, to better analyze the heart rate, a solution for long-term monitoring is required with the desired comfort for subjects. Hence, a development of low-cost heart rate monitoring sensor with a fast response has assumed a great importance.

To date, a number of optical sensors have been demonstrated for heart rate monitoring. Dziuda et al. demonstrated a respiration and cardiac activity by using the fiber Bragg grating (FBG) and showed satisfactory sensing capabilities around $8.3 \mu \mathrm{s}$ with the maximum error $12 \%$ [3]. These sensors are based on the body vibration caused by breathing and the heart activity and affect to the deformation of the FBG when the subject leans on the cushion. Chen et al. [4] performed a study on the fiber optic multimode for the monitoring of the respiratory and heart rate with the accuracy $\pm 2 \mathrm{bpm}$. Yhuwana et al. [5] demonstrated the fiber optic bundle for the measurement of amplitude and frequency of the heart rate signal with a sensitivity about 0.002 $\mathrm{mV} / \mu \mathrm{m}$. The advantage of this system is that the sensing element can be fabricated by using inexpensive components and easily fabricated of the sensor. Życzkowski et al. reported the concept of the modalmetric fiber optic sensor system for human psychophysical activity detection [6]. The sensor comprises a multimode fiber proximately situated in the patient so that time varying acousto-mechanical signals from the patient are coupled by the singlemode optical fiber to a detector.

A fiber optic based sensor system can be used for long-term monitoring as it offers comfort and versatility [7]. Apart from the compact size with the low weight, the fiber optic sensor is immune to electromagnetic fields, water, and corrosion, unlike electrical or chemical devices $[8,9]$. Immunity to electromagnetic fields is a very important feature as a lot of such fields present in the hospital environment [10]. A complete one heartbeat recorded by an electrocardiograph (ECG) can be represented by a P-wave, QRS-complex, and T-wave or shortened by a PQRST-wave. Dziuda et al. reported that the relationship between the anxiety in MRI patients and their RR from the two PQRST-waves acquired by a fibre-optic sensor system [11]. The system presented that non-invasive RR monitoring allows the MRI operator to better control the patient's condition. The $t$-test showed significant differences in changes in anxiety between these groups $\left[t_{(39)}=\right.$ $-2.349, p=0.012$, and Cohen's $d=2.13$ ]. Recently, singlemode-multimode-singlemode (SMS) fibers have been utilized for various sensing applications [12-19]. The SMS fibers have been attracting a greater attention due to their low cost, the freedom in tailoring the output spectrum, and the ease of fabrication.

In this paper, an optical based heart rate monitoring sensor with a low manufacturing cost is demonstrated. The sensor is based on the SMS fiber structure which is spliced at both end of the single mode fiber with a multimode fiber (MMF). An artificial ECG signal produced by a loudspeaker is used to simulate the heart pulse at different rates ranging from $50 \mathrm{bpm}$ to $200 \mathrm{bpm}$. The ECG generator is required to generate an electrical signal. This signal is then fed to an amplified audio amplifier and is used to vibrate the speaker. The loudspeaker is used to convert ECG signals in the electrical form into mechanical signals, and these signals are detected by the SMS fiber. Basically, the proposed heart rate monitoring sensor relies on the vibration change in the loudspeaker which affects the deformation of the sensor probe. The low fabrication cost of the sensor, fast response, and comfort for subjects [20] make our sensor very attractive for the hospital environment. There are numerous studies on the fabrication of optical fiber sensors embedded into the mechanical textile for healthcare monitoring $[21,22]$. To our knowledge, this is the first time that the SMS fiber structure is used as the heart rate monitoring sensor. 


\section{Principles and experimental setup}

An artificial PQRST wave produced by an ECG generator is shown in Fig. 1. The artificial electric signal is translated into the vibration of the mechanical signals through an audio amplifier and a loudspeaker. It is known that the heart function can be determined by the interval and amplitude of the PQRST segment. The atria activation is represented by $\mathrm{P}$ wave, ventricular activation is represented by QRS complex, and ventricular recovery is represented by $\mathrm{T}$ wave $[23,24]$. It is also known that a heart rate in bpm can be determined by counting the number of $\mathrm{RR}$ interval in a minute. Another parameter can be measured to analyze the ECG signal is an RS amplitude and QT interval $[23,24]$.

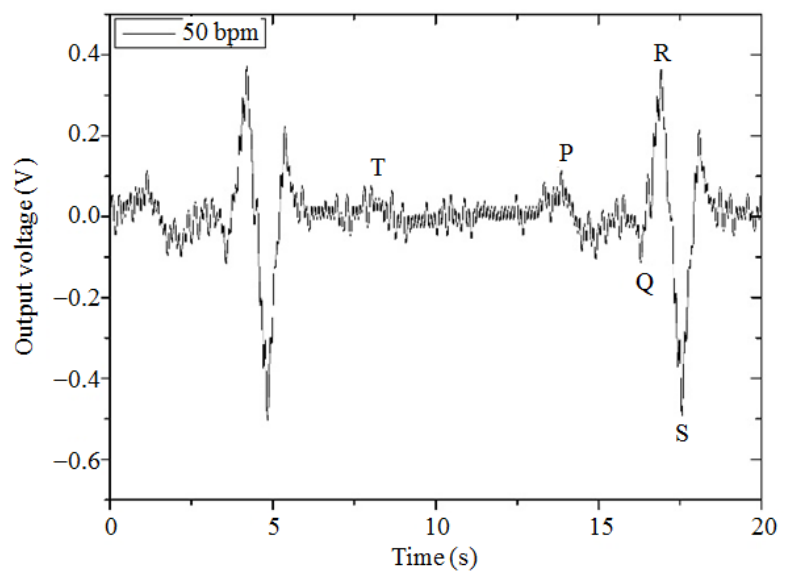

Fig. 1 A typical PQRST wave produced by the ECG generator.

Figure 2(a) shows a schematic diagram of the SMS fiber structure consisting of an MMF section spliced between two singlemode fibers (SMFs). At the first interface between the SMF and MMF, an input light from the SMF will be decomposed and reconstructed by the available guided modes within the MMF. At the first interface between the SMF and MMF (at $z=0$ ), the field profile $\psi(r, 0)$ should have the same profile from the SMF $\phi_{\mathrm{SMF}}(r, 0)$. In the MMF section, there are $m$ available guided modes with the radial field profiles $\phi_{n=1 \text { to } m}(r)$. The field profile $\psi(r, 0)$ can be decomposed by the available guided modes within the MMF as the following equation [10]:

$$
\phi_{\mathrm{SMF}}(r, 0)=\psi(r, 0)=\sum_{n=1}^{n=m} c_{n} \phi_{n}(r)
$$

where $c_{n}$ is the excitation coefficient of each mode, and it is calculated by using an overlap integral between $\phi_{\mathrm{SMF}}(r, 0)$ and $\phi_{n}(r)[10]$ :

$$
c_{n}=\frac{\int_{0}^{\infty} \phi_{\mathrm{SMF}}(r, 0) \phi_{n}(r) r \mathrm{~d} r}{\int_{0}^{\infty} \phi_{n}(r) \phi_{n}(r) r \mathrm{~d} r} .
$$

The interaction between the guided modes creates a destructive or a constructive interference along the MMF section which is called as multimode interference.

In the MMF section, the radial field profile of $\psi(r, z)$ along the propagation distance $z$ can be expressed as [11]

$$
\psi(r, z)=\sum_{n=1}^{n=m} c_{n} \phi_{n} \exp \left(\mathrm{j} \beta_{n} z\right)
$$

where $\beta_{n}$ is the propagation constant of each mode. To obtain an output power at the end of the MMF section, an orthogonal relation between the field profile of the SMF output and the field profile of the MMF at $z$ can be calculated by using [25]

$$
\operatorname{Pout}(z)=10 \lg \left(\left|\sum_{n=1}^{m} c_{n}^{2} \exp \left(\mathrm{j} \beta_{n} z\right)\right|^{2}\right) \text {. }
$$

A fabrication of the SMS fiber structure is carried out by using a commercial fusion splicer. The MMF step index type, with $105 \mu \mathrm{m}$ core diameter and a length of about $10 \mathrm{~mm}$, is connected at both ends to the SMF28 type. For a principal operation of heart rate monitoring by using the SMS fiber structure, an experimental setup is developed and shown in Fig. 2(b). The artificial PQRST wave is generated by the ECG generator to simulate the heart rate at different rates ranging from $50 \mathrm{bpm}$ to $200 \mathrm{bpm}$. The signal is amplified and transferred into mechanical vibration by the audio amplifier and the loudspeaker. The optical light source with a wavelength of $1550 \mathrm{~nm}$ is launched onto the SMS fiber and the MMF placed in the center of the loudspeaker with a tape at the both ends of the MMF with a diameter of $15 \mathrm{~mm}$. The output power from 
the SMS fiber structure is recorded by the optical power meter. Meanwhile, the ECG generator signal is measured by the oscilloscope.

The PQRST signal produced by the generator induces an air pressure variation, which leads to a curvature change on the SMS fiber structure. The curvature creates an applied strain to the SMS fiber structure. It is well known that the applied strain creates a change of the length, core radius and refractive indices of the MMF $[17,18]$. These parameters changes cause the variations of field profile $\psi(r, z)$ and hence the output power $P_{\text {out }}$. Thus, the PQRST signal will change the $P_{\text {out }}$.

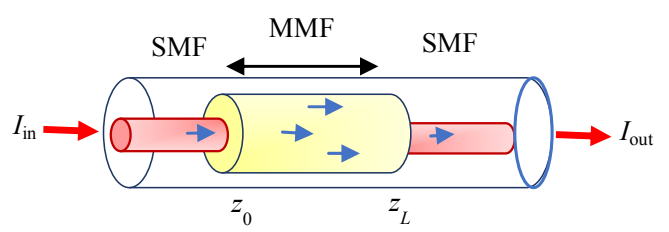

(a)

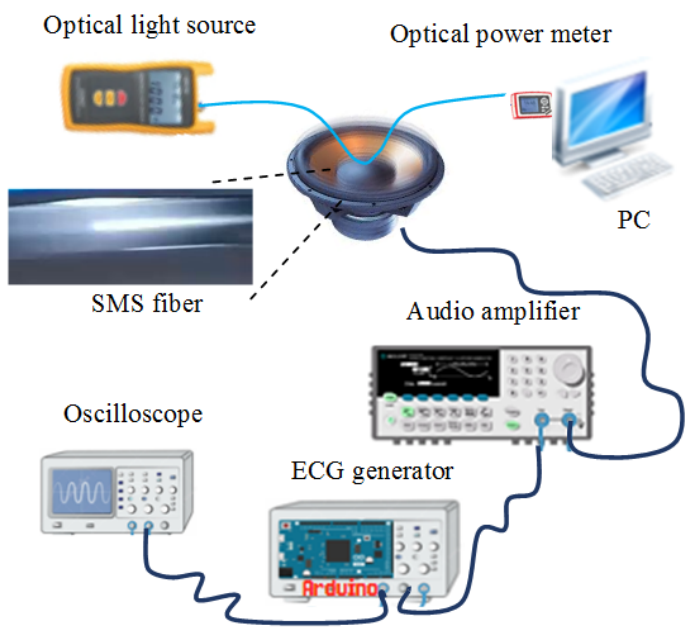

(b)

Fig. 2 A schematic set-up for heart rate monitoring (a) an SMS fiber structure and (b) a schematic of the principal operation of heart rate monitoring with the SMS fiber structure attached to the artificial ECG source.

\section{Results and discussion}

Figure 3 shows the measurement results of optical output power $P_{\text {out }}$ from the SMS fiber structure at different heart rates of $50 \mathrm{bpm}, 85 \mathrm{bpm}$, $100 \mathrm{bpm}$, and $150 \mathrm{bpm}$. It shows the PQRST waves which can be confirmed by comparing it with the output from the oscilloscope. Figures 3(a) - 3(d) show the RS amplitude of changes produced by the heart rate of $50 \mathrm{bpm}, 85 \mathrm{bpm}, 100$ and $150 \mathrm{bpm}$ for about $0.384 \mathrm{dBm}, 0.75 \mathrm{dBm}, 0.87 \mathrm{dBm}$, and 1.64 $\mathrm{dBm}$, respectively. Based on Fig. 3, the suggested SMS fiber sensor system can be used to detect PQRST waves, as well as the RS amplitude.

Figure 4 shows the measured heart rate by the SMS fiber structure compared with the ECG generator. It can be observed that the relation has a linear function with a slope $R^{2}$ of $99.84 \%$, which indicates that the SMS fiber structure can accurately measure the frequency of the heart rate. The results of the experiments show that the sensor has a relative error lower than $1 \%$ as measured heart rate.

Figure 5(a) shows the RR intervals against RS amplitudes for the proposed SMS fiber structure's sensor. The RR interval is to measure the unit of bmp. Since the RR interval increases, the unit of bpm will also increase and need more time to get pushed into the peak and through given by the $\mathrm{R}$ and S events [26, 27]. It is found that RR intervals have a strong correlation with RS amplitudes. The RS interval is determined by the ventricular depolarization. In addition, the QT interval as shown in Fig. 5(b) represents the time taken from the Q event to the $\mathrm{T}$ event compared with the RR interval. The same linear relationship has been proposed in [28]. From Fig. 5(b), it can be observed that the RR interval increases as the QT interval increases and the delayed QT interval may cause a sudden cardiac death [29].

Experimental investigation demonstrates that the SMS fiber structure is capable of detecting the vibration of the pulse signal over a wide frequency ranging from $50 \mathrm{bpm}$ to $200 \mathrm{bpm}$ with a good sensitivity. The resolution of the sensor is obtained by dividing the standard deviation of the readings with the sensitivity of the sensor. Based on the RR 
interval's standard deviation of $0.038 \mathrm{dBm}$, the proposed sensor has a resolution of $2.7 \mathrm{bpm}$. During

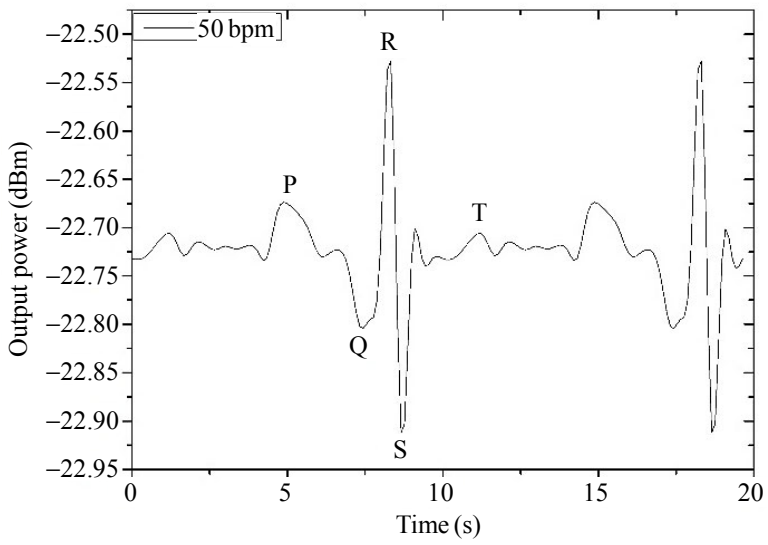

(a)

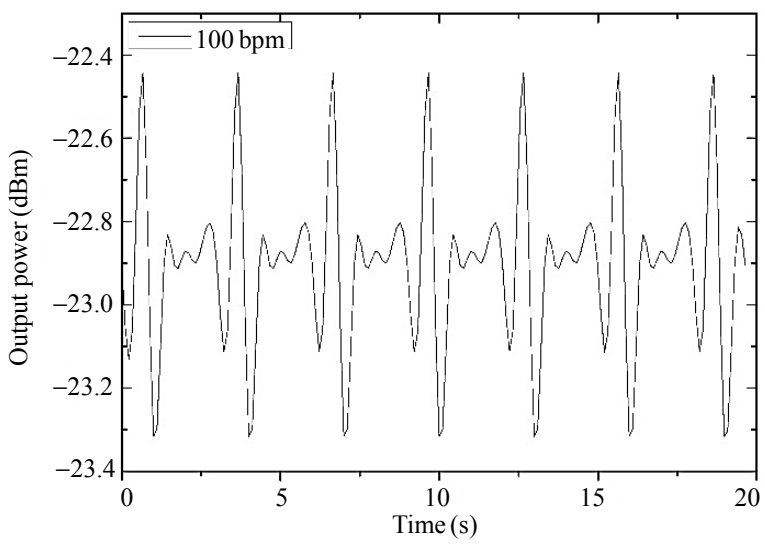

(c) the experiment, the temperature is kept constant around $25^{\circ} \mathrm{C}$.

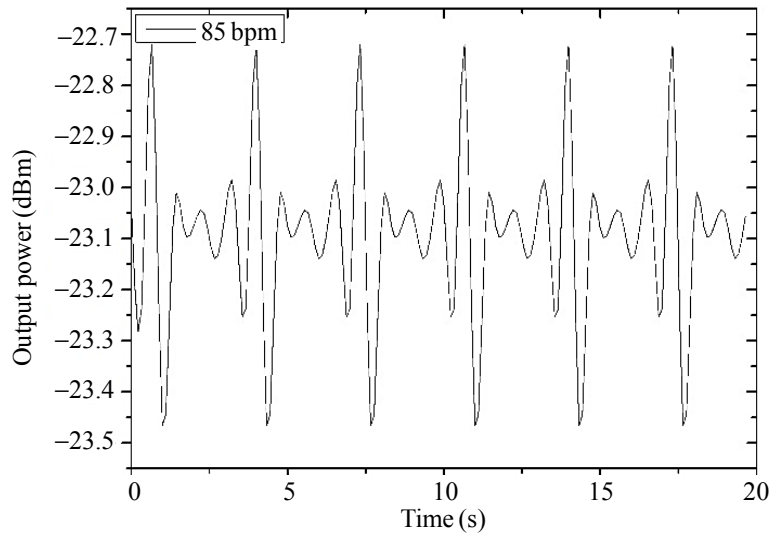

(b)

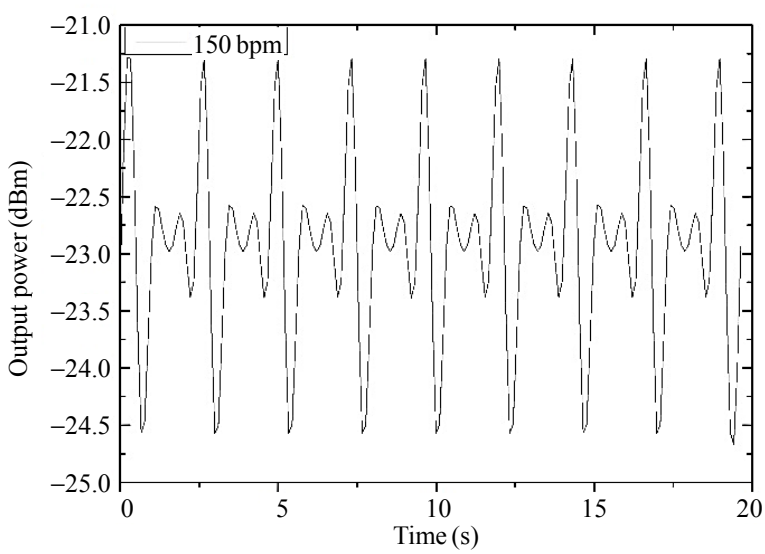

(d)

Fig. 3 Pulse signal from the SMS fiber sensor at different frequencies: (a) $50 \mathrm{bpm}$, (b) $85 \mathrm{bpm}$, (c) $100 \mathrm{bpm}$, and (d) $150 \mathrm{bpm}$.

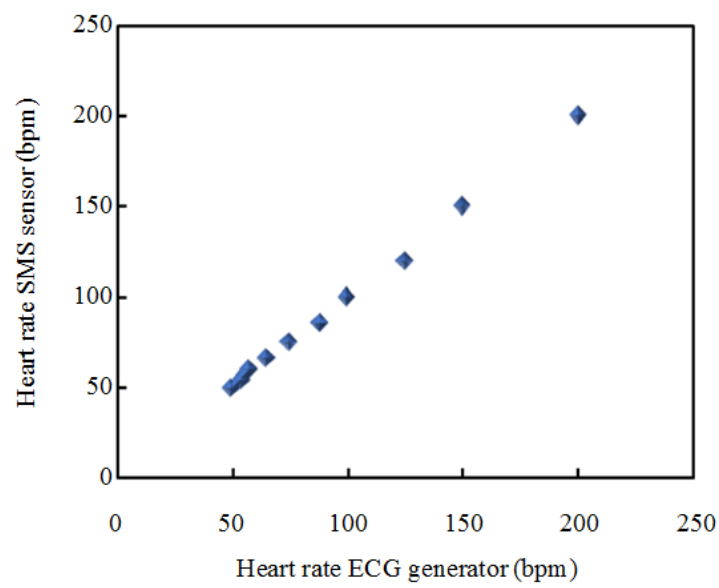

Fig. 4 Heart rate frequency from the ECG generator against the heart rate output frequency from the SMS fiber.

The SMS fiber sensor is compared with some sensors with the new heart rate $[4,5]$. The sensitivity of our sensors is $0.014 \mathrm{dBm} / \mathrm{bpm}$ similar to or higher than those of the other sensors. The SMS fiber sensor is fabricated by using a simple approach and constructed in a straight SMS fiber structure. Chen et al. reported the accuracy $\pm 2 \mathrm{bpm}$ by using multimode fibers. It has been found that the SMS fiber structure with a length of $10 \mathrm{~mm}$ MMF is more sensitive than the plastic optical fiber (POF) based on the displacement sensor with a sensitivity about $0.002 \mathrm{mV} / \mu \mathrm{m}$ for heart rates monitoring as demonstrated by Yuwhana et al.

The main objective of this work is to develop a method of monitoring the heart rate signals by using SMS fiber optic sensors. The proposed sensor is successfully used for monitoring the heart rate 
signals from the artificial ECG. Even though the SMS fiber structure based sensor has advantages, the remaining challenge for practical applications is the detection of the very small signals of the real heart beat. In this work, the test of various models of mechanical signals generated by the ECG generator should be done first before applying it to the human being. Since we want to know the sensitivity respond of the SMS fiber in the mechanical signals generated by the ECG generator. Upon further development, a scheme attaching the SMS fiber structure on micro-sensors and being integrated into mattress would be beneficial for this system, the ballistocardiographic (BCG) signal as reported by Dziuda et al. [30] can be included as the input signal to the loudspeaker as this may provide an optimal signal of the heart beat information for monitoring in the human body with a compact device. The main reason of the experiment is to investigate the ability of the fiber in detecting the heart rate. Further development of the system can be considered to add more variables in the data collection for enhancement such as signals disturbed by sneezing and coughing. The results of this work will be reported in the near future.

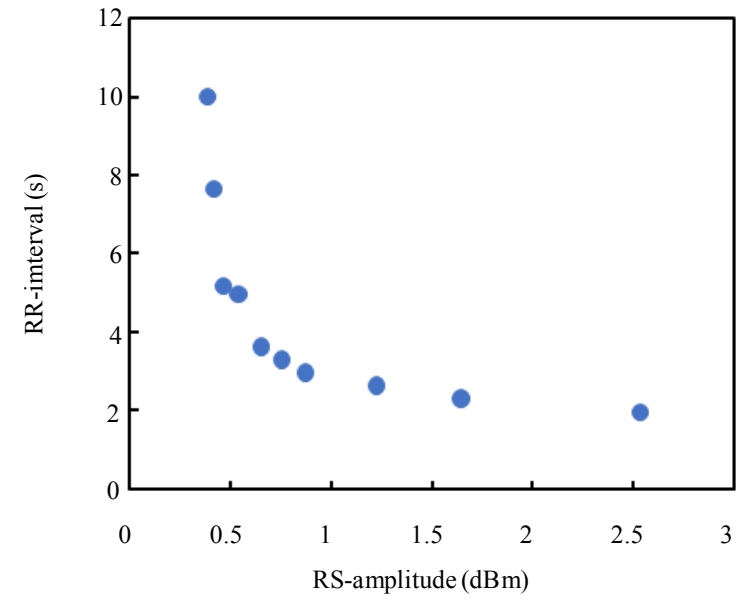

(a)

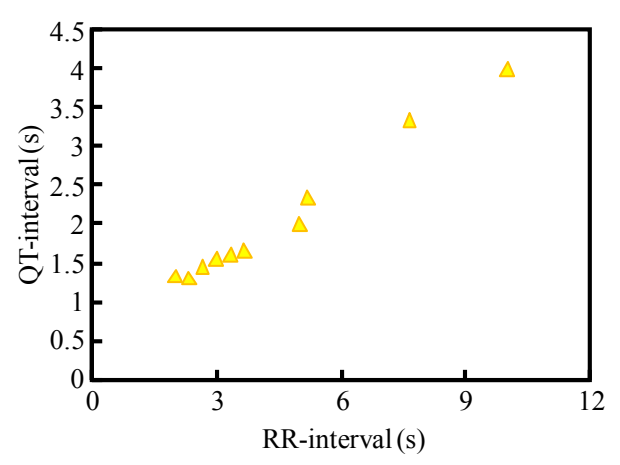

(b)

Fig. 5 Further evaluation on the PQQST wave (a) RR intervals against RS amplitudes for the SMS fiber sensor and (b) RR intervals against QT intervals for the SMS fiber sensor.

\section{Conclusions}

A new heart rate monitoring sensor based on the SMS fiber structure is proposed and experimentally demonstrated. The sensor functions well within the operation range of $50 \mathrm{bpm}$ to $200 \mathrm{bpm}$ with a sensitivity of $0.014 \mathrm{dBm} / \mathrm{bpm}$ under the artificial ECG generator. The SMS optical fiber structure is a suitable candidate for the heart rate monitoring which offers a low fabrication cost of the sensor and a fast response. Further development will be carried out in order to detect the real heart rate in the human body.

\section{Acknowledgment}

This work was supported by the Directorate of Research and Community Service - Ministry of Research, Technology and Higher Education, Republic of Indonesia (Grant Nos. 6/E/KPT/2019 and 954/PKS/ITS/2019).

Open Access This article is distributed under the terms of the Creative Commons Attribution 4.0 International License (http://creativecommons.org/licenses/by/4.0/), which permits unrestricted use, distribution, and reproduction in any medium, provided you give appropriate credit to the original author(s) and the source, provide a link to the Creative Commons license, and indicate if changes were made. 


\section{References}

[1] A. N. Vukadinović, D. Vukadinović, J. Borer, M. Cowie, M. Komajda, M. Lainscak, et al., "Heart rate and its reduction in chronic heart failure and beyond," European Journal of Heart Failure, 2017, 19(10): 1230-1241.

[2] L. G. Lindberg, H. Ugnell, and P. A. Öberg, "Monitoring of respiratory and heart rates using a fibre-optic sensor," Medical and Biological Engineering and Computing, 1992, 30(5): 533-537.

[3] L. Dziuda, F. W. Skibniewski, M. Krej, and J. Lewandowski, "Monitoring respiration and cardiac activity using fiber Bragg grating-based sensor," IEEE Transactions on Biomedical Engineering, 2012, 59(7): 1934-1942.

[4] Z. Chen, D. Lau, J. T. Teo, S. H. Ng, X. Yang, and P. L. Kei, "Simultaneous measurement of breathing rate and heartrate using a microbend multimode fiber optic sensor," Journal of Biomedical Optics, 2014, 19(5): 057001.

[5] Y. Y. Yhuwana, R. Apsari, and M. Yasin, "Fiber optic sensor for heart rate detection," Optik International Journal for Light and Electron Optics, 2017, 134: 28-32.

[6] M. Życzkowski, B. Uzięblo-Zyczkowska, L. Dziuda, and K. Różanowski, "Using modalmetric fiber optic sensors to monitor the activity of the heart," Optical Fibers, Sensors, and Devices for Biomedical Diagnostics and Treatment XI. International Society for Optics and Photonics, San Francisco, Feb. 16, 2011, pp: 789404.

[7] B. Selm, E. A. Gürel, M. Rothmaier, R. M. Rossi, and L. J. Scherer, "Polymeric optical fiber fabrics for illumination and sensorial applications in textiles," Journal of Intelligent Material Systems and Structures, 2010, 21(11): 1061-1071.

[8] T. G. Giallorenzi, J. A. Bucaro, A. Dandridge, G. H. Sigel, J. H. Cole, S. C. Rashleigh, et al., "Optical fiber sensor technology," IEEE Transactions on Microwave Theory and Techniques, 1982, 30(4): 472-511.

[9] S. Liehr, P. Lenke, M. Wendt, K. Krebber, M. Seeger, E. Thiele, et al., "Polymer optical fiber sensors for distributed strain measurement and application in structural health monitoring," IEEE Sensors Journal, 2009, 9(11): 1330-1338.

[10] E. Hanada, "The electromagnetic environment of hospitals: how it is affected by the strength of electromagnetic fields generated both inside and outside the hospital," Annali-Istituto Superiore Di Sanita, 2007, 43(3): 208.

[11] Ł. Dziuda, P. Zieliński, P. Baran, M. Krej, and L. Kopka, "A study of the relationship between the level of anxiety declared by MRI patients in the STAI questionnaire and their respiratory rate acquired by a fibre-optic sensor system," Scientific
Reports, 2019, 9(1): 4341.

[12] Q. Wang, G. Farrell, and W. Yan, "Investigation on single-mode-multimode-single-mode fiber structure", Journal of Lightwave Technology, 2008, 26(5): 512-519.

[13] A. Arifin, A. M. Hatta, M. S. Muntini, and A. Rubiyanto, "Long-range displacement sensor based on SMS fiber structure and OTDR," Photonic Sensors, 2015, 5(2): 166-171.

[14] Q. Wu, A. M. Hatta, P. Wang, Y. Semenova, and G. Farrell, "Use of a bent single SMS fiber structure for simultaneous measurement of displacement and temperature sensing," IEEE Photonics Technology Letters, 2010, 23(2): 130-132.

[15] A. M. Hatta, K. Indriawati, T. Bestariyan, and T. Humada, "SMS fiber structure for temperature measurement using an OTDR," Photonic Sensors, 2013, 3(3): 262-266.

[16] Q. Wu, Y. Semenova, P. Wang, and G. Farrell, "High sensitivity SMS fiber structure based refractometer-analysis and experiment," Optics Express, 2011, 19(9): 7937-7944.

[17] Y. Gong, T. Zhao, Y. J. Rao, and Y. Wu, "All-fiber curvature sensor based on multimode interference," IEEE Photonics Technology Letters, 2011, 23(11): 679-681.

[18] Y. Zhao, X. G. Li, F. C. Meng, and Z. Zhao, "A vibration-sensing system based on SMS fiber structure," Sensors and Actuators A: Physical, 2014, 214: 163-167.

[19] H. Wang, S. Pu, N. Wang, S. Dong, and J. Huang, "Magnetic field sensing based on singlemode-multimode-singlemode fiber structures using magnetic fluids as cladding," Optics Letters, 2013, 38(19): 3765-3768.

[20] Q. Wu, Y. Semenova, P. Wang, and G. Farrell, “The use of a bent singlemode-multimode-singlemode (SMS) fiber structure for vibration sensing," in 21 st International Conference on Optical Fibre Sensors, Ottawa, May 1, 2011, pp: 77535G.

[21] A. Grillet, D. Kinet, J. Witt, M. Schukar, K. Krebber, F. Pirotte, et al., "Optical fiber sensors embedded into medical textiles for healthcare monitoring," IEEE Sensors Journal, 2008, 8(7): 1215-1222.

[22] J. Witt, F. Narbonneau, M. Schukar, K. Krebber, J. De Jonckheere, M. Jeanne, et al., "Medical textiles with embedded fiber optic sensors for monitoring of respiratory movement," IEEE Sensors Journal, 2012, 12 (1): 246-254.

[23] J. Wo, Q. Sun, and H. Liu, "Sensitivity-enhanced fiber optic temperature sensor with strain response suppression," Optical Fiber Technology, 2013, 19(4): 289-292.

[24] Y. Benchaib and M. A. Chikh, "Artificial metaplasiticity MLP results on MIT-BIH cardiac arrhythmias data base," International Journal of 
Advance Research in Computer Engineering \& Technology, 2013, 2(10): 2665-2673.

[25] W. S. Mohammed, P. W. E. Smith, and X. Gu, "All-fibre multimode interference bandpass filter," Optics Letters, 2006, 31(17): 2547-2549.

[26] K. K. Jembula, G. Srinivasulu, and K. S. Prasad, "Design of electrocardiogram (ECG) system on FPGA," International Journal of Engineering and Science, 2013, 3(2): 21-27.

[27] P. E. McSharry, G. D. Clifford, L. Tarassenko, and L. A. Smith, "A dynamical model for generating synthetic electrocardiogram signals," IEEE Transactions on Biomedical Engineering, 2003,
50(3): 289-294.

[28] P. Davey, "A new physiological method for heart rate correction of the QT interval," Heart, 1999, 82(2): 183-186.

[29] P. J. Schwartz and S. Wolf, "Q-T interval as prolongation predictor of sudden death in patients with myocardial infarction," Circulation, 1978, 57(6): 1074-1077.

[30] L. Dziuda and F. W. Skibniewski, “A new approach to ballistocardiographic measurements using fibre Bragg grating-based sensors," Biocybernetics and Biomedical Engineering, 2014, 34(2): 101116. 